\title{
EXISTENCE THEOREMS FOR QUASILINEAR MULTIVALUED BOUNDARY VALUE PROBLEMS IN $\mathbf{R}^{N}$
}

\author{
NIKOLAOS KOUROGENIS and NIKOLAOS S. PAPAGEORGIOU \\ National Technical University, Department of Mathematics, Zografou Campus, Athens 157 80, Greece
}

(Received 9 November, 1998)

\begin{abstract}
In this paper we study quasilinear second order boundary value problems with multivalued right hand side and Dirichlet boundary conditions. We prove three existence theorems. The first two deal with the "convex" and "nonconvex" problems respectively, while the third establishes the existence of extremal solutions. For the first two the proof is based on the theory of nonlinear operators of monotone type, while the proof of the third uses a fixed point argument.
\end{abstract}

1991 Mathematics Subject Classification. 34A60, 34B15.

1. Introduction. In this paper we study quasilinear, second order differential inclusions in $\mathbf{R}^{N}$ with Dirichlet boundary conditions. Problems of this kind for scalar ordinary differential equations were studied by Boccardo-Drabek-GiachettiKucera [3] and Pino-Elgueta-Manasevich [14], using degree theoretic techniques. Here in addition to the vectorial and multivalued character of the problem, we also propose a different approach based on the theory of multivalued operators of monotone type. Moreover, in contrast to the above mentioned works, here the multivalued perturbation term $F$ depends also on the derivative of the unknown function.

After the presentation of some auxiliary results in section 2, in section 3 we prove two existence theorems. The first is for the "convex" problem (i.e. we assume that the multivalued term $F(t, x, y)$ is convex valued) and the second is for the "nonconvex" problem (i.e. we no longer require that $F(t, x, y)$ be convex valued). In section 4 , we replace $F(t, x, y)$ by its extreme points ext $F(t, x, y)$ and look for "extremal solutions". Under some stronger continuity conditions on $F$, we prove that such solutions exist. In contrast to section 3, our method of proof of the result in section 4, is based on a fixed point argument which uses Schauder's fixed point theorem. It appears that our result is the first existence theorem for extremal solutions for quasilinear multivalued boundary value problems.

Our results here extend the semilinear works $(p=2)$ of Frigon-Granas [8], Kravvaritis-Papageorgiou [11], Marano [12] and Pruszko [15] (Theorems 4.5, 4.6 and 4.7). The multivalued problems studied here arise naturally in many applied situations of interest, like control systems with a priori feedback, deterministic systems with uncertainties which are modelled with multifunctions and problems with discontinuous right hand side. This is the case in many problems of mathematical physics and mechanics.

2. Preliminaries. Let $T=[0, b]$ and let $P_{k(c)}\left(\mathbf{R}^{N}\right)=\left\{A \subseteq \mathbf{R}^{N}: A\right.$ is nonempty, compact (and convex) \}. A multifunction $F: T \rightarrow P_{k}\left(\mathbf{R}^{N}\right)$ is said to be "measurable", if for every $v \in \mathbf{R}^{N}$, the $\mathbf{R}_{+}$-valued function $t \rightarrow d(v, F(t))=\inf [\|v-x\|: x \in F(t)]$ is measurable. If on $T$ we consider the Lebesgue $\sigma$-field $\mathcal{L}(T)$, then the above definition 
of measurability is equivalent to saying that $\operatorname{Gr} F=\left\{(t, x) \in T \times \mathbf{R}^{N}: x \in F(t)\right\}$ $\in \mathcal{L}(T) \times B\left(\mathbf{R}^{N}\right)$, where $B\left(\mathbf{R}^{N}\right)$ is the Borel $\sigma$-field of $\mathbf{R}^{N}$ (graph measurability). For $1 \leq p \leq \infty$ we set $S_{F}^{p}=\left\{f \in L^{p}\left(T, \mathbf{R}^{N}\right): f(t) \in F(t)\right.$ a.e. on $\left.T\right\}$. It is easy to see that if $F: T \rightarrow 2^{\mathbf{R}^{N}} \backslash\{\emptyset\}$ is graph measurable, then $S_{F}^{p}$ is nonempty if and only if $t \rightarrow \inf [\|x\|: x \in F(t)] \in L^{p}(T)$. Moreover the set $S_{F}^{p}$ is decomposable; i.e. if $\left(f_{1}, f_{2}, A\right) \in S_{F}^{p} \times S_{F}^{p} \times \mathcal{L}(T)$, then $\chi_{A} f_{1}+\chi_{A^{c}} f_{2} \in S_{F}^{p}$.

If $X$ is a metric space, let $P_{f}(X)=\{A \subseteq X: A$ is nonempty and closed $\}$. On $P_{f}(X)$ we can define a generalized metric, known in the literature as the "Hausdorff metric', by

$$
h(A, B)=\max [\sup (d(a, B): a \in A), \sup (d(b, A): b \in B)]
$$

where $d(a, B)=\inf [d(a, b): b \in B]$ and $d(b, A)=\inf [d(b, a): a \in A]$. It is well known that if $X$ is complete, then so is $\left(P_{f}(X), h\right)$. A multifunction $G: X \rightarrow P_{f}(X)$ is said to be " $h$-continuous", if it is continuous from $X$ into the metric space $\left(P_{f}(X), h\right)$.

Let $V, Y$ be Hausdorff topological spaces. A multifunction $G: V \rightarrow 2^{V} \backslash\{\emptyset\}$ is said to be upper semicontinuous (usc) (resp. lower semicontinuous (lsc)), if for every $C \subseteq Y$ nonempty closed, the set $F^{-}(C)=\{v \in V: F(v) \cap C \neq \emptyset\}$ (resp. $F^{+}(C)=$ $\{v \in V: F(v) \subseteq C\})$ is closed in $V$. If $Y$ is regular and $G: V \rightarrow P_{f}(Y)$ is usc, then $G r G=\{(v, y) \in V \times Y: y \in G(v)\}$ is closed in $V \times Y$ with the product topology. The converse is true if $G(\cdot)$ is locally compact; i.e. for every $v \in V$ there exists $U$ a neighborhood of $v$, such that $\overline{G(U)}$ is compact in $Y$. Also if $V, Y$ are metric spaces, then the above definition of lower semicontinuity of $G: V \rightarrow P_{f}(Y)$, is equivalent to saying that for all $y \in V, v \rightarrow d_{Y}(y, G(v))$ is upper semicontinuous. Also it is equivalent to saying that if $v_{n} \rightarrow v$ in $V$ as $n \rightarrow \infty$, then $G(v) \subseteq \underline{\lim } G\left(v_{n}\right)=$ $\left\{y \in Y: \lim d_{Y}\left(y, G\left(v_{n}\right)\right)=0\right\}=\left\{y \in Y: y=\lim y_{n}, y_{n} \in G\left(v_{n}\right), n \geq 1\right\}$. For details we refer to Klein-Thompson [10].

Next let us recall some definitions and results about multivalued maps of monotone type which we shall need in the sequel. The basic reference is the paper of Browder-Hess [6]. Let $X$ be a reflexive Banach space.

Definition. A multivalued map $A: X \rightarrow 2^{X^{*}}$ is said to be pseudomonotone if

(a) for every $x \in X, A(x)$ is a nonempty, bounded closed and convex subset of $X^{*}$;

(b) $A(\cdot)$ is upper semicontinuous from each finite dimensional subspace $V$ of $X$ into $X^{*}$ furnished with the weak topology;

(c) if $\left\{x_{n}\right\}_{n \geq 1} \subseteq X, x_{n} \stackrel{w}{\rightarrow} x$ and if $x_{n}^{*} \in A\left(x_{n}\right), n \geq 1$, is a sequence such that $\varlimsup \lim \left(x_{n}^{*}, x_{n}-x\right) \leq 0$, then to each element $y \in X$, there exists $x^{*}(y) \in A(x)$ such that $\underline{\lim }\left(x_{n}^{*}, x_{n}-y\right) \geq\left(x^{*}(y), x-y\right)$.

Another closely related notion is as follows.

Definition. A multivalued map $A: X \rightarrow 2^{X^{*}}$ is said to be generalized pseudomonotone if for any sequence $\left\{x_{n}\right\}_{n \geq 1} \subseteq X$ and a corresponding sequence $\left\{x_{n}^{*}\right\}_{n \geq 1} \subseteq X^{*}, \quad x_{n}^{*} \in A\left(x_{n}\right)$, with $x_{n} \stackrel{w}{\rightarrow} x$ in $X, x_{n}^{*} \stackrel{w}{\rightarrow} x^{*}$ in $X^{*}$ and such that $\overline{\lim }\left(x_{n}^{*}, x_{n}-x\right) \leq 0$, then $x^{*} \in A(x)$ and $\left(x_{n}^{*}, x_{n}\right) \rightarrow\left(x^{*}, x\right)$.

A pseudomonotone map is generalized pseudomonotone. The converse is true if $A(\cdot)$ is bounded and for every $x \in X, A(x)$ is nonempty, closed and convex in $X^{*}$. Note that every maximal monotone operator $A: X \rightarrow 2^{X^{*}}$ is generalized pseudomonotone.

In section 4 , we shall use another norm on the classical Banach space $L^{1}\left(T, \mathbf{R}^{N}\right)$, defined by 


$$
\|f\|_{w}=\sup \left[\left\|\int_{t_{1}}^{t_{2}} f(s) d s\right\|: 0 \leq t_{1} \leq t_{2} \leq b\right], \quad f \in L^{p}\left(T, \mathbf{R}^{N}\right) .
$$

This norm is known as the "weak norm" on the space $L^{1}\left(T, \mathbf{R}^{N}\right)$ and the space $L^{1}\left(T, \mathbf{R}^{N}\right)$ furnished with this norm, will be denoted by $L_{w}^{1}\left(T, \mathbf{R}^{N}\right)$. Convergence of a sequence in this weak norm is closely related to the weak convergence of the sequence. The next lemma (which can be found in Kravvaritis-Papageorgiou [11]) illustrates this.

Lemma. If $\left\{u_{n}, u\right\}_{n \geq 1} \subseteq L^{p}\left(T, \mathbf{R}^{N}\right)$ where $1<p<\infty,\left\|u_{n}\right\|_{p} \leq M<\infty$ and $u_{n} \stackrel{\|\cdot\|_{w}}{\rightarrow} u$ as $n \rightarrow \infty$, then $u_{n} \stackrel{w}{\rightarrow} u$ in $L^{p}\left(T, \mathbf{R}^{N}\right)$ as $n \rightarrow \infty$.

Finally in our subsequent considerations we shall need the following basic inequality: $\left(a|a|^{p-2}-b|b|^{p-2}\right)(a-b) \geq 2^{2-p}|a-b|^{p}$ for all $a, b \in \mathbf{R}$ and $p \geq 2$.

3. Existence theorems. Let $T=[0, b]$. The multivalued Dirichlet problem under consideration is the following:

$$
\left\{\begin{array}{l}
-\left(\left\|x^{\prime}(t)\right\|^{p-2} x^{\prime}(t)\right)^{\prime}+F\left(t, x(t), x^{\prime}(t)\right) \ni h(t) \text { a.e. on } T \\
x(0)=x(b)=0, p \geq 2
\end{array}\right\}
$$

Definition. By a solution of problem (1), we mean a function $x \in C^{1}\left(T, \mathbf{R}^{N}\right)$ which satisfies the Dirichlet boundary conditions, $t \rightarrow\left\|x^{\prime}(t)\right\|^{p-2} x^{\prime}(t)$ is absolutely continuous on $T$ and there exists $g \in L^{q}\left(T, \mathbf{R}^{N}\right)\left(\frac{1}{p}+\frac{1}{q}=1\right), g(t) \in F\left(t, x(t), x^{\prime}(t)\right)$ a.e. on $T$ and $-\left(\left\|x^{\prime}(t)\right\|^{p-2} x^{\prime}(t)\right)^{\prime}+g(t)=h(t)$ a.e. on $T$ (recall that by Lebesgue's theorem an absolutely continuous function is differentiable almost everywhere).

In this section we prove two existence theorems for (1), using an approach which is based on the theory of pseudomonotone operators. The first existence theorem assumes that $F(t, x, y)$ has convex values, while the second existence theorem deals with the "nonconvex" problem. We start with the "convex" result. For this purpose our hypotheses on the multifunction $F$ are as follows.

$\mathbf{H}(\mathbf{F})_{\mathbf{1}}: F: T \times \mathbf{R}^{N} \times \mathbf{R}^{N} \rightarrow P_{f c}\left(\mathbf{R}^{N}\right)$ is a multifunction such that

(i) for every $x, y \in \mathbf{R}^{N}, t \rightarrow F(t, x, y)$ is measurable;

(ii) for almost all $t \in T, \operatorname{GrF}(t, \cdot, \cdot)$ is closed;

(iii) for almost all $t \in T$ and all $x, y \in \mathbf{R}^{N},|F(t, x, y)|=\sup \{\|v\|: v \in F(t, x, y)\} \leq$ $\gamma_{1}(t,\|x\|)+\gamma_{2}(t,\|x\|)\|y\|^{p-1}$ where $\sup \left[\gamma_{1}(t,\|x\|):\|x\| \leq r\right] \leq \xi_{1 r}(t)$ a.e. on $T$, $\xi_{1 r} \in L^{q}(T)$ and $\sup \left[\gamma_{2}(t,\|x\|):\|x\| \leq r\right] \leq \xi_{2 r}(t)$ a.e. on $T, \xi_{2 r} \in L^{\infty}(T)$; and

(iv) for almost all $t \in T$, all $x, y \in \mathbf{R}^{N}$ and all $v \in F(t, x, y)$, we have $(v, x)_{\mathbf{R}^{N}} \geq$ $-c\|x\|^{p}-\beta\|y\|^{r}-a(t)$, where $c, \beta \geq 0, r<p, a \in L^{1}(T)$ and $c<\theta_{1}^{p}$ with $\theta_{1}$ being the first eigenvalue of the eigenvalue problem $-\left(\left\|x^{\prime}(t)\right\|^{p-2} x^{\prime}(t)\right)^{\prime}=$ $\theta\|x(t)\|^{p-2} x(t)$ a.e. on $T, x(0)=x(b)=0$.

Theorem 1. If hypotheses $H(F)_{1}$ hold and $h \in L^{q}\left(T, \boldsymbol{R}^{N}\right)$, then problem (1) has a nonempty solution set which is compact in $C\left(T, \boldsymbol{R}^{N}\right)$. 
Proof. Let $A: W_{0}^{1, p}\left(T, \mathbf{R}^{N}\right) \rightarrow W^{-1, q}\left(T, \mathbf{R}^{N}\right)$ be the nonlinear operator defined by

$$
<A(x), y>=\int_{0}^{b}\left\|x^{\prime}(t)\right\|^{p-2}\left(x^{\prime}(t), y^{\prime}(t)\right)_{\mathbf{R}^{N}} d t, \quad x, y \in W_{0}^{1, p}\left(T, \mathbf{R}^{N}\right) .
$$

Here by $<\cdot, \cdot\rangle$ we denote the duality brackets for the pair $\left(W_{0}^{1, p}\left(T, \mathbf{R}^{N}\right)\right.$, $\left.W^{-1, q}\left(T, \mathbf{R}^{N}\right)\right)$. We claim that $A(\cdot)$ is strictly monotone and continuous. Indeed for $x, y \in W_{0}^{1, p}\left(T, \mathbf{R}^{N}\right)$, we have

$$
\begin{aligned}
<A(x)-A(y), x-y>= & \int_{0}^{b}\left\|x^{\prime}(t)\right\|^{p-2}\left(x^{\prime}(t), x^{\prime}(t)-y^{\prime}(t)\right)_{\mathbf{R}^{N}} d t \\
& -\int_{0}^{b}\left\|y^{\prime}(t)\right\|^{p-2}\left(y^{\prime}(t), x^{\prime}(t)-y^{\prime}(t)\right)_{\mathbf{R}^{N}} d t \\
\geq & \int_{0}^{b}\left(\left\|x^{\prime}(t)\right\|^{p}+\left\|y^{\prime}(t)\right\|^{p}-\left\|x^{\prime}(t)\right\|^{p-1}\left\|y^{\prime}(t)\right\|\right. \\
& \left.-\left\|x^{\prime}(t)\right\|\left\|y^{\prime}(t)\right\|^{p-1}\right) d t .
\end{aligned}
$$

Note that $\left\|x^{\prime}(\cdot)\right\|^{p-1},\left\|y^{\prime}(\cdot)\right\|^{p-1} \in L^{q}(T)$. Via Hölder's inequality, we obtain

$$
\begin{aligned}
<A(x)-A(y), x-y> & \geq\left\|x^{\prime}\right\|_{p}^{p}+\left\|y^{\prime}\right\|_{p}^{p}-\left\|x^{\prime}\right\|_{p}^{p-1}\left\|y^{\prime}\right\|_{p}-\left\|x^{\prime}\right\|_{p}\left\|y^{\prime}\right\|_{p}^{p-1} \\
& =\left\|x^{\prime}\right\|_{p}^{p-1}\left(\left\|x^{\prime}\right\|_{p}-\left\|y^{\prime}\right\|_{p}\right)-\left\|y^{\prime}\right\|_{p}^{p-1}\left(\left\|x^{\prime}\right\|_{p}-\left\|y^{\prime}\right\|_{p}\right) \\
& =\left(\left\|x^{\prime}\right\|_{p}^{p-1}-\left\|y^{\prime}\right\|_{p}^{p-1}\right)\left(\left\|x^{\prime}\right\|_{p}-\left\|y^{\prime}\right\|_{p}\right) .
\end{aligned}
$$

Using the basic inequality mentioned at the end of section 2, we have

$$
<A(x)-A(y), x-y>\geq c \int_{0}^{b}\left|\left\|x^{\prime}(t)\right\|-\left\|y^{\prime}(t)\right\|\right|^{p} d t \text { for some } c>0 .
$$

If $<A(x)-A(y), x-y>=0$, then $\left\|x^{\prime}(t)\right\|=\left\|y^{\prime}(t)\right\|$ a.e. on $T$. Hence using this fact in the equality

$$
\begin{aligned}
<A(x)-A(y), x-y>= & \int_{0}^{b}\left\|x^{\prime}(t)\right\|^{p-2}\left(x^{\prime}(t), x^{\prime}(t)-y^{\prime}(t)\right)_{\mathbf{R}^{N}} d t \\
& -\int_{0}^{b}\left\|y^{\prime}(t)\right\|^{p-2}\left(y^{\prime}(t), x^{\prime}(t)-y^{\prime}(t)\right)_{\mathbf{R}^{N}} d t=0
\end{aligned}
$$

we obtain $\int_{0}^{b}\left\|x^{\prime}(t)\right\|^{p-2}\left\|x^{\prime}(t)-y^{\prime}(t)\right\|^{2} d t=0$. From this last equality it follows that $\left\|x^{\prime}(t)\right\|=0$ a.e. on $T$ or $\left\|x^{\prime}(t)-y^{\prime}(t)\right\|=0$ a.e. on $T$. In the first case, we have $x^{\prime}(t)=y^{\prime}(t)=0$ a.e. on $T$ and in the second case $x^{\prime}(t)=y^{\prime}(t)$ a.e. on $T$. Since $x, y \in W_{0}^{1, p}\left(T, \mathbf{R}^{N}\right)$, we have $x(t)=\int_{0}^{t} x^{\prime}(s) d s=\int_{0}^{t} y^{\prime}(s) d s=y(t)$ for all $t \in T$. Now $x=y$ and this proves the strict monotonicity of the map $A(\cdot)$. To prove the continuity of $A(\cdot)$, let $x_{n} \rightarrow x$ in $W_{0}^{1, p}\left(T, \mathbf{R}^{N}\right)$; then if by $\|\cdot\|$ we denote the norm of $W_{0}^{1, p}\left(T, \mathbf{R}^{N}\right)$, and by $\|\cdot\|_{*}$ the norm of $W^{-1, q}\left(T, \mathbf{R}^{N}\right)$, we have 


$$
\begin{aligned}
\left\|A\left(x_{n}\right)-A(x)\right\|_{*} & =\sup \left[<A\left(x_{n}\right)-A(x), y>:\|y\| \leq 1\right] \\
& =\sup \left[\int_{0}^{b}\left(\left\|x_{n}^{\prime}(t)\right\|^{p-2} x_{n}^{\prime}(t)-\left\|x^{\prime}(t)\right\|^{p-2} x^{\prime}(t), y^{\prime}(t)\right)_{\mathbf{R}^{N}} d t:\|y\| \leq 1\right] \\
& \leq\|\| x_{n}^{\prime}\left\|^{p-2} x_{n}^{\prime}-\right\| x^{\prime}\left\|^{p-2} x^{\prime}\right\|_{q} \text { (Hölder's inequality). }
\end{aligned}
$$

Invoking the extended Lebesgue convergence theorem (see Ash [1], Theorem 7.5.2, p. 295), we have $\left\|A\left(x_{n}\right)-A(x)\right\|_{*} \rightarrow 0$ as $n \rightarrow \infty$. $A(\cdot)$ is as claimed strictly monotone and continuous, with domain all of $W_{0}^{1, p}\left(T, \mathbf{R}^{N}\right)$. Thus $A(\cdot)$ is maximal monotone and also pseudomonotone (see Browder-Hess [6, proposition 8, p. 266]).

Next let $G: W_{0}^{1, p}\left(T, \mathbf{R}^{N}\right) \rightarrow 2^{L^{q}\left(T, \mathbf{R}^{N}\right)}$ be the multifunction defined by

$$
G(x)=S_{F\left(\cdot, x(\cdot), x^{\prime}(\cdot)\right)}^{q}=\left\{f \in L^{q}\left(T, \mathbf{R}^{N}\right): f(t) \in F\left(t, x(t), x^{\prime}(t)\right) \text { a.e. on } T\right\} .
$$

From Lemma 3.3 of Kandilakis-Papageorgiou [9], we know that $G(\cdot)$ has nonempty, closed, convex values, is usc into $L^{q}\left(T, \mathbf{R}^{N}\right)$ furnished with the weak topology (denoted by $L^{q}\left(T, \mathbf{R}^{N}\right)_{w}$ ) and is bounded.

Then let $H(x)=A(x)+G(x)$. We claim that $x \rightarrow H(x)$ is pseudomonotone from $W_{0}^{1, p}\left(T, \mathbf{R}^{N}\right)$ into $2^{W^{-1, q}\left(T, \mathbf{R}^{N}\right)} \backslash\{\emptyset\}$. Note that $H(\cdot)$ has nonempty, closed and convex values and is bounded. To show that $H(\cdot)$ is pseudomonotone, it suffices to prove that it is generalized pseudomonotone. To this end let $\left\{\left(x_{n}, v_{n}\right)\right\}_{n \geq 1} \subseteq G r H$ and assume that $x_{n} \stackrel{w}{\rightarrow} x$ in $W_{0}^{1, p}\left(T, \mathbf{R}^{N}\right), v_{n} \stackrel{w}{\rightarrow} v$ in $W^{-1, q}\left(T, \mathbf{R}^{N}\right)$ and $\stackrel{\lim }{v_{1}}<v_{n}, x_{n}-x>$ $\leq 0$. We have $v_{n}=A\left(x_{n}\right)+g_{n}$ with $g_{n} \in G\left(x_{n}\right), n \geq 1$. Note that, by virtue of hypothesis $H(F)_{1}$ (iii), we have

$$
\left\|g_{n}(t)\right\| \leq \gamma_{1}\left(t, M_{1}\right)+\gamma_{2}\left(t, M_{1}\right)\left\|x_{n}^{\prime}(t)\right\|^{p-1} \text { a.e. on } T,
$$

where $M_{1}=\sup _{n \geq 1}\left\|x_{n}\right\|_{\infty}<\infty \quad$ (recall that $W_{0}^{1, p}\left(T, \mathbf{R}^{N}\right)$ embeds continuously in $C\left(T, \mathbf{R}^{N}\right)$ ). From (3) and hypothesis $H(F)_{1}$ (iii) it follows that $\left\{g_{n}\right\}_{n \geq 1} \subseteq L^{q}\left(T, \mathbf{R}^{N}\right)$ is bounded and so by passing to a subsequence if necessary, we may assume that $g_{n} \stackrel{w}{\rightarrow} g$ in $L^{q}\left(T, \mathbf{R}^{N}\right)$ as $n \rightarrow \infty$. Recalling that $W_{0}^{1, p}\left(T, \mathbf{R}^{N}\right)$ embeds compactly in $L^{p}\left(T, \mathbf{R}^{N}\right)$ (see Brezis [5]), we have that $x_{n} \rightarrow x$ in $L^{p}\left(T, \mathbf{R}^{N}\right)$. We have

$$
<g_{n}, x_{n}-x>=\int_{0}^{b}\left(g_{n}(t), x_{n}(t)-x(t)\right)_{\mathbf{R}^{N}} d t \rightarrow 0 \text { as } n \rightarrow \infty .
$$

Since $\overline{\lim }<v_{n}, x_{n}-x>\leq 0$, it follows that

$$
\varlimsup<A\left(x_{n}\right), x_{n}-x>\leq 0 .
$$

But we have already seen that $A(\cdot)$ is pseudomonotone and hence generalized pseudomonotone. Now $A\left(x_{n}\right) \stackrel{w}{\rightarrow} A(x)$ in $W^{-1, q}\left(T, \mathbf{R}^{N}\right)$ and $<A\left(x_{n}\right), x_{n}>\rightarrow<A(x), x>$ as $n \rightarrow \infty$. But note that $<A\left(x_{n}\right), x_{n}>=\left\|x_{n}^{\prime}\right\|_{p}^{p}, n \geq 1$, and $<A(x), x>=\left\|x_{w}^{\prime}\right\|_{p}^{p}$. We have $\left\|x_{n}^{\prime}\right\|_{p} \rightarrow\left\|x^{\prime}\right\|_{p}$ as $n \rightarrow \infty$. On the other hand, we know that $x_{n}^{\prime} \stackrel{w}{\rightarrow} x^{\prime}$ in $L^{p}\left(T, \mathbf{R}^{N}\right)$ (since $x_{n} \stackrel{w}{\rightarrow} x$ in $\left.W_{0}^{1, p}\left(T, \mathbf{R}^{N}\right)\right)$. Since $L^{p}\left(T, \mathbf{R}^{N}\right)$ has the Kadec-Klee property, we infer that $x_{n}^{\prime} \rightarrow x^{\prime}$ in $L^{p}\left(T, \mathbf{R}^{N}\right)$. Now $x_{n} \rightarrow x$ in $W_{0}^{1, p}\left(T, \mathbf{R}^{N}\right)$ and thus, by passing to a subsequence if necessary, we may also assume that $x_{n}^{\prime}(t) \rightarrow x^{\prime}(t)$ a.e. on 
$T$ and $x_{n}(t) \rightarrow x(t)$ for all $t \in T$. Invoking Theorem 3.1 of Papageorgiou [13], we obtain

$$
g(t) \in \overline{\operatorname{conv}} \varlimsup\left\{g_{n}(t)\right\}_{n \geq 1} \subseteq \overline{\operatorname{conv}} \varlimsup \overline{l i m} F\left(t, x_{n}(t), x_{n}^{\prime}(t)\right) \subseteq F\left(t, x(t), x^{\prime}(t)\right) \text { a.e. on } T
$$

the last inclusion following from hypothesis $H(F)_{1}$ (ii). Finally we have $v=A(x)+g$, with $g \in G(x)$ and $<v_{n}, x_{n}>\rightarrow<v, x>$ as $n \rightarrow \infty$. Therefore $H(\cdot)$ is pseudomonotone.

Next we shall show that $H(\cdot)$ is coercive. For $g \in G(x)$ and $v=A(x)+g \in H(x)$ we have

$$
\begin{aligned}
<v, x> & =<A(x), x>+<g, x> \\
& \geq\left\|x^{\prime}\right\|_{p}^{p}-c\|x\|_{p}^{p}-\beta\|x\|_{r}^{r}-\|a\|_{1} \quad\left(\text { see hypothesis } H(F)_{1}\right. \text { (iv)) }
\end{aligned}
$$

Recall that $\|x\|_{p} \leq \frac{1}{\theta_{1}}\left\|x^{\prime}\right\|_{p}$ for all $x \in W_{0}^{1, p}\left(T, \mathbf{R}^{N}\right.$ ) (see Boccardo et al. [3]). Thus we have

$$
<v, x>\geq\left\|x^{\prime}\right\|_{p}^{p}-\frac{c}{\theta_{1}^{p}}\left\|x^{\prime}\right\|_{p}^{p}-\widehat{\beta}\|x\|_{p}^{r}-\|a\|_{1} \quad\left(\widehat{\beta}=\beta b^{\frac{1}{r}-\frac{1}{p}}\right)
$$

By hypothesis $H(F)_{1}$ (iv), $1-\frac{c}{\theta^{p}}>0$. Recalling that $\left\|x^{\prime}\right\|_{p}$ is an equivalent norm for $W_{0}^{1, p}\left(T, \mathbf{R}^{N}\right)$, from (4) we infer at once that $H(\cdot)$ is coercive. But a bounded, coercive, pseudomonotone operator is surjective (see Browder-Hess [6], Theorem 3). Therefore we can find $x \in W_{0}^{1, p}\left(T, \mathbf{R}^{N}\right)$ and $g \in G(x)$ such that

$$
\begin{aligned}
& <A(x), y>+<g, y>=<h, y>\text { for all } y \in W_{0}^{1, p}\left(T, \mathbf{R}^{N}\right) \\
& \Rightarrow \int_{0}^{b}\left\|x^{\prime}(t)\right\|^{p-2}\left(x^{\prime}(t), y^{\prime}(t)\right)_{\mathbf{R}^{N}} d t=\int_{0}^{b}(h(t)-g(t), y(t))_{\mathbf{R}^{N}} d t \text { for all } y \in W_{0}^{1, p}\left(T, \mathbf{R}^{N}\right) .
\end{aligned}
$$

From (5) it follows that $\left\|x^{\prime}(\cdot)\right\|^{p-2} x^{\prime}(\cdot) \in W^{1, q}\left(T, \mathbf{R}^{N}\right)$ and so $t \rightarrow\left\|x^{\prime}(t)\right\|^{p-2} x^{\prime}(t)$ is absolutely continuous on $T$. Since the function $u \rightarrow \phi_{p}(u)=\|u\|^{p-2} u$ is strictly increasing, we have that $x^{\prime} \in C\left(T, \mathbf{R}^{N}\right)$ and so $x \in C^{1}\left(T, \mathbf{R}^{N}\right)$. Moreover, if by $(\cdot, \cdot)_{p q}$ we denote the duality brackets for the pair $\left(L^{p}\left(T, \mathbf{R}^{N}\right), L^{q}\left(T, \mathbf{R}^{N}\right)\right)$, from (5) we have $(A(x), y)_{p q}=(h-g, y)_{p q}$ for all $y \in W_{0}^{1, p}\left(T, \mathbf{R}^{N}\right)$. Since $W_{0}^{1, p}\left(T, \mathbf{R}^{N}\right)$ is dense in $L^{p}\left(T, \mathbf{R}^{N}\right)$, we deduce that

$$
\left\{\begin{array}{l}
-\left(\left\|x^{\prime}(t)\right\|^{p-2} x^{\prime}(t)\right)^{\prime}+g(t)=h(t) \text { a.e. on } T, \\
x(0)=x(b)=0 .
\end{array}\right\}
$$

with $g(t) \in F\left(t, x(t), x^{\prime}(t)\right)$ a.e. on $T$. Hence $x(\cdot)$ is a solution of (1).

We claim that the solution set of (1) is bounded in $W_{0}^{1, p}\left(T, \mathbf{R}^{N}\right)$. Indeed as above using hypothesis $H(F)_{1}$ (iv) and the fact $\|x\|_{p} \leq \frac{1}{\theta_{1}}\left\|x^{\prime}\right\|_{p}$, for all $x \in W_{0}^{1, p}\left(T, \mathbf{R}^{N}\right)$, we have

$$
\frac{\left(1-\frac{c}{\theta_{1}^{p}}\right)\left\|x^{\prime}\right\|_{p}^{p}-\widehat{\beta}\|x\|_{p}^{r}-\|a\|_{1}}{\left\|x^{\prime}\right\|_{p}} \leq \frac{1}{\theta_{1}}\|h\|_{q}
$$


from which it follows at once that the solution set of (1) is bounded in $W_{0}^{1, p}\left(T, \mathbf{R}^{N}\right)$. Since $W_{0}^{1, p}\left(T, \mathbf{R}^{N}\right)$ embeds compactly in $C\left(T, \mathbf{R}^{N}\right)$ (see Brezis [5]), we see that the solution set is relatively compact in $C\left(T, \mathbf{R}^{N}\right)$. In fact arguing as above, via the pseudomonotonicity of the operator $A(\cdot)$ and the Kadec-Klee property of $L^{p}\left(T, \mathbf{R}^{N}\right)$, it follows easily that the solution set of (1) is $C\left(T, \mathbf{R}^{N}\right)$-closed; hence $C\left(T, \mathbf{R}^{N}\right)$-compact.

By suitably modifying the above proof, we can also have an existence theorem for the nonconvex problem. The hypotheses on the multifunction $F(t, x, y)$ are the following.

$\mathbf{H}(\mathbf{F})_{2}: F: T \times \mathbf{R}^{N} \times \mathbf{R}^{N} \rightarrow P_{k}\left(\mathbf{R}^{N}\right)$ is a multifunction such that

(i) $(t, x, y) \rightarrow F(t, x, y)$ is graph measurable;

(ii) for almost all $t \in T,(x, y) \rightarrow F(t, x, y)$ is lsc;

(iii) for almost all $t \in T$ and all $x, y \in \mathbf{R}^{N},|F(t, x, y)|=\sup [\|v\|: v \in F(t, x, y)] \leq$ $\gamma_{1}(t,\|x\|)+\gamma_{2}(t,\|x\|)\|y\|$ where $\sup \left[\gamma_{1}(t,\|x\|):\|x\| \leq r\right] \leq \xi_{1 r}(t)$ a.e. on $T$, $\xi_{1 r} \in L^{q}(T)$ and $\sup \left[\gamma_{2}(t,\|x\|):\|x\| \leq r\right] \leq \xi_{2 r}(t)$ a.e. on $T, \xi_{2 r} \in L^{\infty}(T)$; and

(iv) for almost all $t \in T$, all $x, y \in \mathbf{R}^{N}$ and all $v \in F(t, x, y),(v, x)_{\mathbf{R}^{N}} \geq$ $-c\|x\|^{p}-\beta\|y\|^{r}-a(t)$, where $c, \beta \geq 0, r<p, a \in L^{1}(T)$ and $c<\theta_{1}^{p}$ with $\theta_{1}$ being the first eigenvalue of the eigenvalue problem $-\left(\left\|x^{\prime}(t)\right\|^{p-2} x^{\prime}(t)\right)^{\prime}=$ $\theta\|x(t)\|^{p-2} x(t)$ a.e. on $T, x(0)=x(b)=0$.

THeOrem 2. If hypotheses $H(F)_{2}$ hold and $h \in L^{q}\left(T, \boldsymbol{R}^{N}\right)$, then problem (1) has at least one solution.

Proof. Let $G: W_{0}^{1, p}\left(T, \mathbf{R}^{N}\right) \rightarrow P_{f}\left(L^{q}\left(T, \mathbf{R}^{N}\right)\right)$ be defined by

$$
G(x)=S_{F\left(\cdot, x(\cdot), x^{\prime}(\cdot)\right)}^{q}=\left\{f \in L^{q}\left(T, \mathbf{R}^{N}\right): f(t) \in F\left(t, x(t), x^{\prime}(t)\right) \text { a.e. on } T\right\} .
$$

From Lemma 4 of Kandilakis-Papageorgiou [9], we know that $G(\cdot)$ is 1sc, bounded and has decomposable values. Apply Theorem 3 of Bressan-Colombo [4], to obtain $r: W_{0}^{1, p}\left(T, \mathbf{R}^{N}\right) \rightarrow L^{q}\left(T, \mathbf{R}^{N}\right)$ a continuous map such that $r(x) \in G(x)$ for all $x \in W_{0}^{1, p}\left(T, \mathbf{R}^{N}\right)$. Arguing as in the proof of Theorem 2, we can show that $x \rightarrow A(x)+r(x)$ is bounded, pseudomonotone and coercive, hence is surjective. Therefore we can find $x \in W_{0}^{1, p}\left(T, \mathbf{R}^{N}\right)$ such that

$$
<A(x), y>+<r(x), y>=<h, y>\text { for all } y \in W_{0}^{1, p}\left(T, \mathbf{R}^{N}\right) .
$$

From the proof of Theorem 2, we know that the above equality implies that $x(\cdot)$ is a solution of (1).

4. Extremal solutions. In this section, we turn our attention to the problem of existence of extremal solutions. More specifically, we consider the following quasilinear multivalued Dirichlet problem:

$$
\left\{\begin{array}{l}
-\left(\left\|x^{\prime}(t)\right\|^{p-2} x^{\prime}(t)\right)^{\prime}+\operatorname{ext} F\left(t, x(t), x^{\prime}(t)\right) \ni h(t) \text { a.e. on } T, \\
x(0)=x(b)=0 .
\end{array}\right\}
$$


Here by $\operatorname{ext} F(t, x, y)$ we denote the extreme points of the set $F(t, x, y)$. Note that $\operatorname{ext} F(t, x, y)$ need not be closed and $(x, y) \rightarrow \operatorname{ext} F(t, x, y)$ need not have any continuity properties, even if $F(t, \cdot, \cdot)$ is regular enough. So the resolution of problem (6) cannot be derived as a special case of Theorems 2 or 3 . We need a new approach. In fact our method here will be based on a fixed point argument which uses Schauder's fixed point theorem, instead of the theory of operators of monotone type. This allows us to drop the one sided growth condition $H(F)_{1}$ (iv). However we have to restrict a little our growth condition on $x$ and also strengthen the continuity hypothesis on $F(t, \cdot, \cdot)$. In the literature there is only one other result on the existence of extremal solutions for second order multivalued Dirichlet problems. It is due to Kravvaritis-Papageorgiou [11] and concerns the semilinear problem $(p=2)$. Their method of proof is different and uses the Green's function of the operator $\left(-x^{\prime \prime}, H_{0}^{1}\left(T, \mathbf{R}^{N}\right)\right)$. For extremal solutions of first order Cauchy problems in Banach spaces we refer to the elegant work of DeBlasi-Pianigiani [8].

The precise hypotheses on the multifunction $F(t, x, y)$, are the following.

$\mathbf{H}(\mathbf{F})_{3}: F: T \times \mathbf{R}^{N} \times \mathbf{R}^{N} \rightarrow P_{k c}\left(\mathbf{R}^{N}\right)$ is a multifunction such that

(i) for all $x, y \in \mathbf{R}^{N}, t \rightarrow F(t, x, y)$ is measurable;

(ii) for almost all $t \in T,(x, y) \rightarrow F(t, x, y)$ is $h$-continuous;

(iii) for almost all $t \in T$ and all $x, y \in \mathbf{R}^{N},|F(t, x, y)|=\sup [\|v\|: v \in F(t, x, y)] \leq$ $a(t)+c\|x\|^{p-1}+\beta\|y\|^{p-1}$, with $a \in L^{q}(T), c, \beta \geq 0$ and $\frac{c}{\theta_{1}^{p}}+\frac{\beta}{\theta_{1}}<1$.

Theorem 3. If hypotheses $H(F)_{3}$ hold and $h \in L^{q}\left(T, \boldsymbol{R}^{N}\right)$, then problem (6) has at least one solution.

Proof. We shall start by deriving some a priori bounds for $\left\|x^{\prime}\right\|_{p}$ and $\|x\|_{\infty}$, when $x(\cdot)$ is a solution of (6). To this end let $x(\cdot) \in W_{0}^{1, p}\left(T, \mathbf{R}^{N}\right)$ be such a solution. We have

$$
\left\{\begin{array}{l}
-\left(\left\|x^{\prime}(t)\right\|^{p-2} x^{\prime}(t)\right)^{\prime}+g(t)=h(t) \text { a.e. on } T, \\
x(0)=x(b)=0 .
\end{array}\right\}
$$

where $g \in L^{q}\left(T, \mathbf{R}^{N}\right), g(t) \in F\left(t, x(t), x^{\prime}(t)\right)$ a.e. on $T$. Taking the inner product with $x(t)$ and then integrating over $T=[0, b]$, we obtain

$$
\begin{aligned}
& \int_{0}^{b}\left\|x^{\prime}(t)\right\|^{p} d t=\int_{0}^{b}(h(t)-g(t), x(t))_{\mathbf{R}^{N}} d t \leq\|g-h\|_{q}\|x\|_{p} \leq \frac{1}{\theta_{1}}\|g-h\|_{q}\left\|x^{\prime}\right\|_{p} \\
& \Rightarrow\left\|x^{\prime}\right\|_{p}^{p-1} \leq \frac{1}{\theta_{1}}\|g-h\|_{q} \\
& \quad \leq \frac{1}{\theta_{1}}\left(\|a\|_{q}+c\|x\|_{p}^{p-1}+\beta\left\|x^{\prime}\right\|_{p}^{p-1}+\|h\|_{q}\right) \text { (see hypothesis } H(F)_{3} \text { (iii)) } \\
& \Rightarrow\left(1-\frac{c}{\theta_{1}^{p}}-\frac{\beta}{\theta_{1}}\right)\left\|x^{\prime}\right\|_{p}^{p-1} \leq \frac{1}{\theta_{1}}\left(\|a\|_{q}+\|h\|_{q}\right) .
\end{aligned}
$$

Since by hypothesis $H(F)_{3}$ (iii) $\frac{c}{\theta_{1}^{p}}+\frac{\beta}{\theta_{1}}<1$, from the above inequality we obtain that there exists $M_{1}>0$ such that $\left\|x^{\prime}\right\|_{p} \leq M_{1}$ for all solutions $x(\cdot)$ of (6). Since 
$W_{0}^{1, p}\left(T, \mathbf{R}^{N}\right)$ embeds continuously in $C\left(T, \mathbf{R}^{N}\right)$, we can find $\gamma>0$ such that $\|x\|_{\infty} \leq \gamma\left\|x^{\prime}\right\|_{p}$ for all $x \in W_{0}^{1, p}\left(T, \mathbf{R}^{N}\right)$. So there exists $M_{2}>0$ such that $\|x\|_{\infty} \leq M_{2}$ for all solutions $x(\cdot)$ of (6). Also directly from the inclusion we see that $\left\{\left\|x^{\prime}\right\|^{p-2} x^{\prime}\right\} \subseteq W^{1, q}\left(T, \mathbb{R}^{N}\right)$ is bounded and independent of $x$. Hence it is bounded in $C\left(T, \mathbb{R}^{N}\right)$ independent of $x$ and we can find $M_{3}>0$ such that $\left\|x^{\prime}(t)\right\| \leq M_{3}$ for all $t \in T$ and all solutions $x(\cdot)$ of (6). Having obtained these bounds, we see that in what follows we may replace $F(t, x, y)$ by $\widehat{F}(t, x, y)=F\left(t, p_{M_{2}}(x), p_{M_{3}}(y)\right)$, where for every $M>0, p_{M}$ denotes the $M$-radial retraction on $\mathbf{R}^{N}$; i.e. $p_{M}(x)=\left\{\begin{array}{ll}x & \text { if }\|x\| \leq M \\ \frac{M x}{\|x\|} & \text { if }\|x\|>M\end{array}\right.$. Then we see that $|\widehat{F}(t, x, y)|=\sup [\|v\|: v \in \widehat{F}(t, x, y)] \leq a(t)+c M_{2}^{p-1}+\beta M_{3}^{p-1}=$ $\psi(t)$ a.e. on $T$, with $\psi \in L^{q}(T)$.

Let $V=\left\{u \in L^{q}\left(T, \mathbf{R}^{N}\right):\|u(t)\| \leq \psi(t)\right.$ a.e. on $\left.T\right\}$ and let $\eta: V \rightarrow W_{0}^{1, p}\left(T, \mathbf{R}^{N}\right)$ be the map which to each $u \in V$ assigns the unique solution of the boundary value problem

$$
\left\{\begin{array}{l}
-\left(\left\|x^{\prime}(t)\right\|^{p-2} x^{\prime}(t)\right)^{\prime}=u(t)+h(t) \text { a.e. on } T \\
x(0)=x(b)=0
\end{array}\right\}
$$

Taking the inner product with $x(t)$ and then integrating over $T=[0, b]$, we obtain

$$
\begin{aligned}
\left\|x^{\prime}\right\|_{p}^{p} & \leq\|u+h\|_{q}\|x\|_{p} \leq \frac{1}{\theta_{1}}\|u+h\|_{q}\left\|x^{\prime}\right\|_{p} \\
\Rightarrow\left\|x^{\prime}\right\|_{p} & \leq\left[\frac{1}{\theta_{1}}\left(\|u\|_{q}+\|h\|_{q}\right)\right]^{\frac{1}{p-1}}=M_{4} .
\end{aligned}
$$

Let $M=\max \left\{M_{1}+b^{\frac{1}{p}} M_{2}, M_{4}\right\}$ and set $K=\bar{B}(0, M)=$ the closed $M$-ball in $W_{0}^{1, p}\left(T, \mathbf{R}^{N}\right)$. Note that $K$ is a bounded, closed, convex subset of $W_{0}^{1, p}\left(T, \mathbf{R}^{N}\right)$, hence is $w$-compact. Since $W_{0}^{1, p}\left(T, \mathbf{R}^{N}\right)$ embeds compactly in $C\left(T, \mathbf{R}^{N}\right), K$ is relatively compact in $C\left(T, \mathbf{R}^{N}\right)$. Moreover, since the embedding is weakly continuous, $K$ is weakly compact, convex in $C\left(T, \mathbf{R}^{N}\right)$, therefore it is closed in $C\left(T, \mathbf{R}^{N}\right)$, hence compact (in fact note that the weak topology on each norm bounded subset of $W_{0}^{1, p}\left(T, \mathbf{R}^{N}\right)$ is induced by the metric of $\left.C\left(T, \mathbf{R}^{N}\right)\right)$.

Let $G_{1}: K \rightarrow P_{f c}\left(L^{1}\left(T, \mathbf{R}^{N}\right)\right)$ be defined by

$$
G_{1}(x)=h-S_{\widehat{F}\left(\cdot, x(\cdot), x^{\prime}(\cdot)\right)}^{1}=h-\left\{f \in L^{1}\left(T, \mathbf{R}^{N}\right): f(t) \in \widehat{F}\left(t, x(t), x^{\prime}(t)\right) \text { a.e. on } T\right\} .
$$

Apply Theorem 5.1 of Tolstonogov [16] to produce $g_{1}: K \rightarrow L_{w}^{1}\left(T, \mathbf{R}^{N}\right)$ continuous, such that $g_{1}(x) \in \operatorname{ext} G_{1}(x)$ for all $x \in K$. Recall that $L_{w}^{1}\left(T, \mathbf{R}^{N}\right)$ denotes the Banach space $L^{1}\left(T, \mathbf{R}^{N}\right)$ equipped with the "weak norm" $\|f\|_{w}=\sup \left[\left\|\int_{t_{1}}^{t_{2}} f(s) d s\right\|: 0 \leq t_{1} \leq t_{2} \leq b\right]$ and on $K$ we consider the $C\left(T, \mathbf{R}^{N}\right)$-topology which as we already mentioned coincides with the weak $W_{0}^{1, p}\left(T, \mathbf{R}^{N}\right)$-topology. From Benamara [2] we know that $\operatorname{ext} G_{1}(x)=\operatorname{ext}\left(h-S_{\hat{F}\left(\cdot, x(\cdot), x^{\prime}(\cdot)\right)}^{1}\right)=h-S_{\text {ext } \hat{F}\left(\cdot, x(\cdot), x^{\prime}(\cdot)\right)}^{1}$. We extend $g_{1}(\cdot)$ on all of $W_{0}^{1, p}\left(T, \mathbf{R}^{N}\right)$, by setting $\widehat{g}_{1}=g_{1} \circ p_{K}$, where $p_{K}$ is the metric projection on $K$ in the space $W_{0}^{1, p}\left(T, \mathbf{R}^{N}\right)$. It is well-known that $p_{K}(\cdot)$ is continuous. So $\widehat{g}_{1}: W_{0}^{1, p}\left(T, \mathbf{R}^{N}\right) \rightarrow$ $L_{w}^{1}\left(T, \mathbf{R}^{N}\right)$ is continuous and we have $\widehat{g}_{1}(x) \in h-S_{\text {ext } \hat{F}\left(\cdot, p_{K}(x)(\cdot), p_{K}(x)^{\prime}(\cdot)\right)}$. Recall from the proof of Theorem 2 that $A: W_{0}^{1, p}\left(T, \mathbf{R}^{N}\right) \rightarrow W^{-1, q}\left(T, \mathbf{R}^{N}\right)$ is maximal monotone, 
strictly monotone and since $\langle A(x), x\rangle=\left\|x^{\prime}\right\|_{p}^{p}$, it is also coercive. Thus $A(\cdot)$ is surjective. Then $A^{-1}: W^{-1, q}\left(T, \mathbf{R}^{N}\right) \rightarrow W_{0}^{1, p}\left(T, \mathbf{R}^{N}\right)$ is well-defined and clearly is continuous. Since $|\widehat{F}(t, x, y)| \leq \psi(t)$ a.e. on $T$ and $\psi \in L^{q}(T)$, we have that $\widehat{g}_{1}(x) \in L^{q}\left(T, \mathbf{R}^{N}\right)$ for every $x \in W_{0}^{1, p}\left(T, \mathbf{R}^{N}\right)$. Since $L^{q}\left(T, \mathbf{R}^{N}\right)=L^{p}\left(T, \mathbf{R}^{N}\right)^{*}$ embeds compactly in $W^{-1, q}\left(T, \mathbf{R}^{N}\right)=W_{0}^{1, p}\left(T, \mathbf{R}^{N}\right)^{*}$ using the lemma from section 2 , we have that $\widehat{g}_{1} \circ A^{-1}: \quad W^{-1, q}\left(T, \mathbf{R}^{N}\right) \rightarrow W^{-1, q}\left(T, \mathbf{R}^{N}\right)$ is continuous. Note that $\left(\widehat{g}_{1} \circ A^{-1}\right)\left(W^{-1, q}\left(T, \mathbf{R}^{N}\right)\right)=\widehat{g}_{1}\left(W_{0}^{1, p}\left(T, \mathbf{R}^{N}\right)\right)=g_{1}(K)$ which is compact in $W^{-1, q}\left(T, \mathbf{R}^{N}\right)$, since it is weakly compact in $L^{q}\left(T, \mathbf{R}^{N}\right)$ and the latter embeds compactly in $W^{-1, q}\left(T, \mathbf{R}^{N}\right)$. Thus we can apply Schauder's fixed point theorem and obtain $v \in W^{-1, q}\left(T, \mathbf{R}^{N}\right)$ such that $v=\widehat{g}_{1}\left(A^{-1}(v)\right)$. Let $x=A^{-1}(v) \in W_{0}^{1, p}\left(T, \mathbf{R}^{N}\right)$. Then we have

$$
A(x)=\widehat{g}_{1}(x) \in h-S_{\text {ext }}^{q} \widehat{F\left(\cdot, p_{K}(x)(\cdot), p_{K}(x)^{\prime}(\cdot)\right)}
$$

and from the choice of $M>0$ and the definition of $K$, we have $x \in K$. Hence

$$
A(x)=g_{1}(x) \in h-S_{\operatorname{ext} \widehat{F}\left(\cdot, x(\cdot), x^{\prime}(\cdot)\right)}^{q}
$$

Hence there exists $f \in S_{\operatorname{ext}}^{q} \widehat{F\left(\cdot, x(\cdot), x^{\prime}(\cdot)\right)}$ such that

$$
\begin{aligned}
& <A(x), y>=<h-f, y>\text { for all } y \in W_{0}^{1, p}\left(T, \mathbf{R}^{N}\right) \\
& \Rightarrow \int_{0}^{b}\left\|x^{\prime}(t)\right\|^{p-2}\left(x^{\prime}(t), y^{\prime}(t)\right)_{\mathbf{R}^{N}} d t=\int_{0}^{b}(h(t)-f(t), y(t))_{\mathbf{R}^{N}} d t \text { for all } y \in W_{0}^{1, p}\left(T, \mathbf{R}^{N}\right) .
\end{aligned}
$$

Then as we did in the proof of Theorem 1, we deduce that $x(\cdot)$ is a solution of (6).

\section{REFERENCES}

1. R. Ash, Real analysis and probability (Academic Press, 1972).

2. M. Benamara, Points extremaux, multiapplications et fonctionelles integrales, These de 3eme cycle (Université de Grenoble, 1975).

3. L. Boccardo, P. Drabek, D. Giachetti and M. Kučera, Generalization of Fredholm alternative for nonlinear differential operators, Nonlinear Anal. 10 (1986), 1083-1103.

4. A. Bressan and G. Colombo, Extensions and selections of maps with decomposable values, Studia Math. 90 (1988), 69-85.

5. H. Brezis, Analyse fonctionelle (Masson, Paris, 1983).

6. F. Browder and P. Hess, Nonlinear mappings of monotone type, J. Funct. Anal. 11 (1972), 251-294.

7. F. S. DeBlasi and G. Pianigiani, Nonconvex valued differential inclusions in Banach spaces, J. Math. Anal. Appl. 157 (1991), 469-494.

8. M. Frigon and A. Granas, Theoremes d'existence pour des inclusions differentielles sans convexes, C. R. Acad. Sci. Paris Sér. I Math. 310 (1990), 819-822.

9. D. Kandilakis and N. S. Papageorgiou, Existence theorems for nonlinear boundary value problems for second order differential inclusions, J. Differential Equations 132 (1996), $107-125$.

10. E. Klein and A. Thompson, Theory of correspondences (Wiley, 1984).

11. D. Kravvaritis and N.S. Papageorgiou, Boundary value problems for nonconvex differential inclusions, J. Math. Anal. Appl. 185 (1994), 146-160.

12. S. Marano, Existence theorems for a multivalued boundary value problem, Bull. Austr. Math. Soc. 45 (1992), 249-260. 
13. N. S. Papageorgiou, Convergence theorems for Banach space valued integrable multifunctions, Intern. J. Math and Math. Sci. 10 (1987), 433-442.

14. M. D. Pino, M. Elgueta and R. Manasevich, A homotopic deformation along $p$ of a Leray-Schauder degree result and existence for $\left(\left|u^{\prime}\right|^{p-2} u^{\prime}\right)^{\prime}+f(t, u)=0, u(0)=u(T)=0, J$. Differential Equations 80 (1989), 1-13.

15. T. Pruszko, Some applications of the topological degree theory to multivalued boundary value problem, Dissertationes Math., 229 (1984).

16. A. Tolstonogov, Extreme continuous selectors of multivalued maps and their applications, J. Differential Equations 122 (1995), 161-180. 